\title{
The Effect of Supplementing the Diet with Purple Garlic (Allium sativum) on Productivity Variables and Intestinal Morphometry in Broilers
}

D. Vargas ${ }^{1}$, C. Rosario², B. Hernández¹, S.I. Peña ${ }^{1}$, M.T. Casaubon²,

S.C. Carlin ${ }^{3}$, M.E. Juárez ${ }^{4}, M$. Martínez ${ }^{3}$, I. Juárez ${ }^{3}$

10.18805/IJAR.BF-1450

\section{ABSTRACT}

Background: Garlic is considered a natural growth promoter in animals, but the effects on the productive performance of broilers are variable. Therefore, the impact on growth performance and intestinal morphometry were evaluated to know the impact of using different levels of purple garlic in the broilers' diet.

Methods: Clinically healthy 1-day-old Cobb Vantress 500 broilers ( $n=98$, with an equal number of two sex) were randomized into four dietary treatment groups. The birds of the $T_{0}$ group were fed with a conventional diet, while the $T_{1}, T_{2}$ and $T_{3}$ groups were fed with diets supplemented with $0.1,0.2$ and $0.3 \%$ of purple garlic.

Result: The body weight gain (BWG) of the fattening female birds did not differ significantly $(P<0.05)$ compared to the control. The BWG and the total benefit at $T_{2}$ and $T_{3}$ were considerably higher in males at 40 days. The duodenum showed that the values of the mean height of the villi were significantly $(P<0.05)$ lower in $T_{1}$ and $T_{2}$ than in the other treatments. Purple garlic has a significant effect as a growth promoter in male broilers, supplemented at a higher rate of supplementation, i.e., 0.2 and $0.3 \%$ levels.

Key words: Additive, Antibiotic growth promoter, Broiler, Intestinal morphometry, Natural feed supplement.

\section{INTRODUCTION}

The global livestock industry faces challenges to achieve higher productivity due to the increasing demand for animal protein. Increasing the human population with a ban on antibiotic growth promoters requires alternatives in the form of functional foods, nutraceuticals, or phytochemicals. Garlic (Allium sativum) has been reported helpful for poultry production due to its physiological, antimicrobial, energizing and hepatoprotective effects; it stimulates the immune, digestive and growth promoter systems (Al-Shuwaili et al., 2015).

The Spanish ecotype "Morado de Las Pedroñeras" is a crop in constant use for human consumption and has been used in animals for some years in Europe, it has been shown to have an antibiotic and anticancer effect due to its high level of thiosulfinate and allicin, which are the active principles of garlic (Pérez-Ortiz et al., 2020). However, the impact of garlic on the productive variables in chickens is variable gender-wise (Lee et al., 2017). Therefore, this study aims to determine the effect of supplementation with Spanish purple garlic on the productive indicators related to growth in females and males since, to our knowledge, there are no studies that have evaluated it.

\section{MATERIALS AND METHODS}

The experiment was carried out at the Veterinary Medicine Faculty, National Autonomous University of Mexico, Mexico City, during the first semester of 2020. The Institutional Subcommittee approved all procedures of this study (protocol number 094) to compliance with the care and use
1Department of Physiology and Pharmacology, Faculty of Veterinary Medicine and Zootechnics, National Autonomous University of Mexico, Mexico City, P.C. 04510, Mexico.

${ }^{2}$ Department of Birds Medicine and Zootechnics, Faculty of Veterinary Medicine and Zootechnics, National Autonomous University of Mexico, Mexico City, P.C. 04510, Mexico.

${ }^{3}$ Department of Preventive Medicine and Public Health, Faculty of Veterinary Medicine and Zootechnics, National Autonomous University of Mexico, Mexico City, P.C. 04510, Mexico.

${ }^{4}$ Department of Animal Nutrition, National Institute of Medical Sciences and Nutrition Salvador Zubiran, Mexico City, P.C.14080, Mexico.

Corresponding Author: D. Vargas, Department of Physiology and Pharmacology, Faculty of Veterinary Medicine and Zootechnics, National Autonomous University of Mexico, Mexico City, P.C. 04510 , Mexico. Email: dinorahvestrada@fmvz.unam.mx

How to cite this article: Vargas, D., Rosario, C., Hernández, B., Peña, S.I., Casaubon, M.T., Carlin, S.C., Juárez, M.E., Martínez, M. and Juárez, I. (2022). The Effect of Supplementing the Diet with Purple Garlic (Allium sativum) on Productivity Variables and Intestinal Morphometry in Broilers. Indian Journal of Animal Research. DOI: 10.18805/IJAR.BF-1450.

Submitted: 05-10-2021 Accepted: 25-12-2021 Online: 14-02-2022

of animals in poultry production and according to the international recommendations (Meluzzi and Sirri, 2009).

\section{Experimental birds and diets}

From 1 day of age, ninety-eight clinically healthy broiler chicks Cobb Vantress 500 were distributed with an utterly 
randomized design ( $50 \%$ female and $50 \%$ male) with average body weight $39.5 \pm 0.84 \mathrm{~g}$, into four dietary treatments groups having three repetitions for each treatment (6/7 birds).

Zooallium ${ }^{\circledR}[Z]$, a product standardized to $65 \%$ purple garlic ecotype from Las Pedroñeras, Cuenca, Spain, was used in this study. Birds were fed either conventional $\left(T_{0}\right)$ diets or supplemented diets $\left(T_{1}\right.$ to $\left.T_{3}\right)$ with $0.1,0.2$ and $0.3 \%$ purple garlic. Birds were reared in vertical battery cages within the experimental isolation unit during 40 days of growth and kept under uniform management conditions.

All the birds were weighed weekly in the morning before feeding and watering until they were 40 days old. The performance of the experimental birds in terms of body weight gain (BWG), feed intake $(\mathrm{FI})$, average daily weight gain (ADG), feed conversion ratio (FCR) and total profit (TP). All variables were recorded by sex (female and male birds).

\section{Gut morphometry}

At the end of the experiment, three birds were randomly selected per group and sacrificed by cervical dislocation (EFSA, 2019) to study intestinal morphometry. Jejunum and duodenum sections were collected and fixed in $10 \%$ formalin. Histological sections were stained with hematoxylin and eosin staining. Ten randomly selected villi were measured on each slide per microscopic field from each section. To measure the length of the villi, eyepiece lenses $(10 X)$ and objective lenses $(3.2 X)$ were used to measure the width of the villi. The lengths of the villi were measured from the tip of the villi to the level of the bottom of the villus crypt (depth). Villus width was defined as the distance from the outer epithelial border to the exterior of the opposite epithelial border along a line passing through the vertical midpoint of the villi. Intestinal morphometry was only performed in female bird samples.

\section{Statistical analysis}

The sample size calculation was carried out using published data of Chicken bodyweight considering $\alpha$ and $\beta$ values of 0.05 and 0.80 , determined on the $\mathrm{JMP} \circledast 10$ program. Productive variables were analyzed using PROC GLM (version 9.3; SAS Ins. Inc., Cary, NC, USA), morphometry data were analyzed using ANOVA. The Tukey-Kramer test was used for specific contrasts in all analyses mentioned above. The normality of the variable and the homogeneity of the variations between the groups were previously evaluated to analyze the results. The criteria for statistical significance were $\mathrm{P}<0.05$.

\section{RESULTS AND DISCUSSION}

Effect of garlic on the productive variables of chickens

In this study, the potential effect of the diet with concentrate supplemented with different doses of purple garlic on the growth promotion and intestinal morphometry of female and male Cobb Vantress 500 broilers were evaluated. Broilers supplemented for 40 days with $0.2 \%$ and $0.3 \%$ of garlic reached a weight significative at the end of this period, a weight significative $(P<0.05)$ more significant than that achieved with the other groups, however, there was no significant difference in feed conversion among four groups. The effect of feeding different levels of purple garlic powder on the performance of broilers is shown in Table 1. The productive parameters did not differ significantly $(P<0.05)$ among four groups in females. However, the BWG and TP in the $T_{2}$ and $T_{3}$ groups in males were considerably higher than the other groups. At the numerical level, the productive parameters: ADG and FCR were better in the $T_{2}$ and $T_{3}$ groups than in the other groups (Table 1).

According to what has been published, the effect of garlic on the productive variables of chickens is variable: Choi et al. (2010), who administered to poultry for 35 days, with $1 \%, 3 \%, 5 \%$ garlic powder, did not find a significant effect on productive parameters; instead, Javandel et al. (2008) used diets supplemented with garlic flour and only in the highest dose (2\%), they observe an increase in feed conversion. With such small amounts of $0.2 \%$ and $0.3 \%$ of purple garlic, the present study achieved the goal of achieving a higher weight than the groups that did not receive a garlic supplement. In the works of Puvaca et al. (2014), garlic improved the feed conversion rate with $0.5 \%$ garlic powder in the diet of broiler chicken. With a dose of $5 \%$ garlic, Al-Shuwaili et al. (2015) reported improved live bird weight and feed conversion when administered for eight weeks. On the other hand, Karangiya et al. (2016) concluded that a $1 \%$ dose of garlic works as a growth promoter in chickens. With a lower amount of purple garlic from "Las Pedroñeras", the present work achieved this objective, so a low-medium dose was able to improve the productive variables.

\section{The female bird's body weight}

Did not differ significantly on any recorded day. However, the body weight in the males of the $T_{2}$ and $T_{3}$ groups was significantly higher $(P<0.05)$ on day 40 compared to the other groups (Table 2).

\section{The body weight in the male broilers}

Was significantly lower $(P<0.05)$ in the control and $T_{1}$ groups than in other groups and the female groups. Chicken genders have been documented to affect glycan and lipid metabolism, related to growth performance and differentsex hormones generally influence metabolic processes (Varlamov et al. 2015). In chickens, like other species, there is an effect of sex on the growth rate; absolute sexual dimorphism is reached in chickens at 14 days of age, from which point its value increases in the males. In this early growth stage, corresponding to the pre-inflection phase of the growth curve, bodyweight shows an exponentially increasing pattern. Male and female broilers have different initial growth potentials; for example, males use food more efficiently than females, from week 5 to 8 . This explains why the males reached greater weight at day 40 than the females in groups $T_{2}$ and $T_{3}$ not so in groups $T_{0}$ that before day 40 
The Effect of Supplementing the Diet with Purple Garlic (Allium sativum) on Productivity Variables and Intestinal Morphometry...

the males showed a greater weight than the females, however at day 40 , they were $6 \%$ less heavy than females. In the $T_{1}$ group from day 2 to 40 , the females were heavier than the males.

The available information shows that garlic can improve production parameters. However, according to the available literature, the different results obtained with the administration of garlic could be due to a difference in the administered dose, the ecotype of the garlic, the sex of the chicken.

\section{Gut morphometry}

The statistical analysis of the morphometry of the duodenum showed that values of mean villi height were significantly $(P<0.05)$ lower in $T_{1}$ and $T_{2}$ compared with other groups. The values of duodenum mean villi width were no differences between the groups. The jejunum values of mean villi height in $T_{1}$ were lower than the other groups, the mean $T_{3}$ group values were significantly $(P<0.05)$ lower than the $T_{2}$ group. (Fig 1). Four experimental groups observed a non-significant difference was observed in the total intestine, duodenum, jejunum and ileum lengths among four experimental groups (Fig 2).

Duodenum is the first part of the small intestine where $95 \%$ of the fat is digested; in the jejunum, the major nutrients are digested and absorbed (Svihus, 2014). In the current study, the statistical analysis of the morphometry showed that the values of the mean height of the villi of the duodenum were significantly $(P<0.05)$ lower in $T_{1}$ and $T_{2}$ than in the other groups. Furthermore, the jejunum values of the mean height of the villi in $T_{1}$ were lower than in the other groups; the mean jejunal villus width values in all treatment groups were significantly $(P<0.05)$ lower than in the control group. Therefore, villi height and width have been positively related to increased nutrient uptake (Oladele et al. 2012) or compensatory mechanism. The morphometry of the villi is reported to change during chick development and the greatest dimension of the villi is observed at 20-22 days (Arce et al. 2008). Unfortunately, in the present study, it was impossible to sample the chickens at this stage and could it be done until the end of the study (40 days) and only carried out in female birds. The present work supports the use of purple garlic from Las Pedroñeras, Spain (standardized

Table 1: Effect of feeding different levels of purple garlic on growth performance of broiler chicks for 42 days.

\begin{tabular}{lccccc}
\hline Parameters $(\mathrm{g})$ & $\mathrm{T}_{0}$ & $\mathrm{~T}_{1}$ & $\mathrm{~T}_{2}$ & $\mathrm{~T}_{3}$ & $\mathrm{SEM}$ \\
\hline ADG (F) & 61.9 & 62.7 & 62.4 & 61.5 & 0.188 \\
ADG (M) & 58.3 & 59.8 & 68.9 & 69.0 & 2.178 \\
BWG (F) & 2476.4 & 2506.1 & 2495.7 & 2461.3 & 7.544 \\
BWG (M) & $2331.2^{\mathrm{A}}$ & $2393.2^{\mathrm{A}}$ & $2756.4^{\mathrm{B}}$ & $2761.5^{\mathrm{B}}$ & 87.108 \\
TFI (F) & $3524.3^{\mathrm{A}}$ & $3718.9^{\mathrm{A}}$ & $3563.6^{\mathrm{A}}$ & $3913.1^{\mathrm{B}}$ & 32.064 \\
TFI (M) & 3563.2 & 3697.5 & 3767.6 & 3679.5 & 29.013 \\
FCR (F) & 1.42 & 1.48 & 1.43 & 1.59 & 0.029 \\
FCR (M) & 1.53 & 1.54 & 1.37 & 1.33 & 0.041 \\
TP at 40 d (F) & 2421.4 & 2447.9 & 2438.7 & 2403.5 & 7.406 \\
TP at 40 d (M) & $2275.6^{\mathrm{A}}$ & $2337.7^{\mathrm{A}}$ & $2701.9^{\mathrm{B}}$ & $2708.1^{\mathrm{B}}$ & 87.468 \\
\hline F: Fema
\end{tabular}

F: Female birds; M: Male birds; Means on the same row with the same superscripts are not significantly different (P>0.05). Body weight gain (BWG); Total Feed intake (TFI); Average daily gain (ADG); Feed conversion ratio (FCR); Total profit (TP).

Table 2: Chicken body weight (grams) fed different levels of purple garlic.

\begin{tabular}{|c|c|c|c|c|c|}
\hline Day & $\mathrm{T}_{0}$ & $T_{1}$ & $T_{2}$ & $T_{3}$ & SEM \\
\hline $2(F)$ & 54.9 & 58.2 & 57.04 & 57.8 & 0.542 \\
\hline $2(\mathrm{M})$ & 55.6 & 55.5 & 54.4 & 53.4 & 0.395 \\
\hline $7(F)$ & 139.5 & 144.7 & 151.01 & 153.7 & 2.414 \\
\hline 7 (M) & 141.2 & 141.4 & 142.3 & 139.3 & 0.479 \\
\hline $14(F)$ & 361.5 & 369.6 & 378 & 369.8 & 2.546 \\
\hline $14(\mathrm{M})$ & 367.4 & 343 & 385.7 & 349.1 & 7.291 \\
\hline $21(F)$ & 719.0 & 778.1 & 784.3 & 771.01 & 11.267 \\
\hline $21(\mathrm{M})$ & 800.5 & 703.9 & 835.2 & 769.6 & 21.087 \\
\hline $28(F)$ & 1279.9 & 1309.9 & 1311.6 & 1295 & 5.599 \\
\hline $28(\mathrm{M})$ & 1332.5 & 1223.6 & 1400.2 & 1335.1 & 27.669 \\
\hline $35(F)$ & 1893.3 & 1924.1 & 1937.3 & 1890.9 & 8.674 \\
\hline $35(\mathrm{M})$ & 1972.1 & 1868.2 & 2050.2 & 1988.3 & 28.564 \\
\hline $40(\mathrm{~F})$ & 2476.4 & 2506.1 & 2495.7 & 2461.3 & 7.544 \\
\hline $40(\mathrm{M})$ & $2331.2^{\mathrm{A}}$ & $2393.2^{A}$ & $2756.4^{B}$ & $2761.5^{B}$ & 87.108 \\
\hline
\end{tabular}

F: Female birds; M: Male birds; Means on the same raw with the same superscripts are not significantly different $(P>0.05)$. 


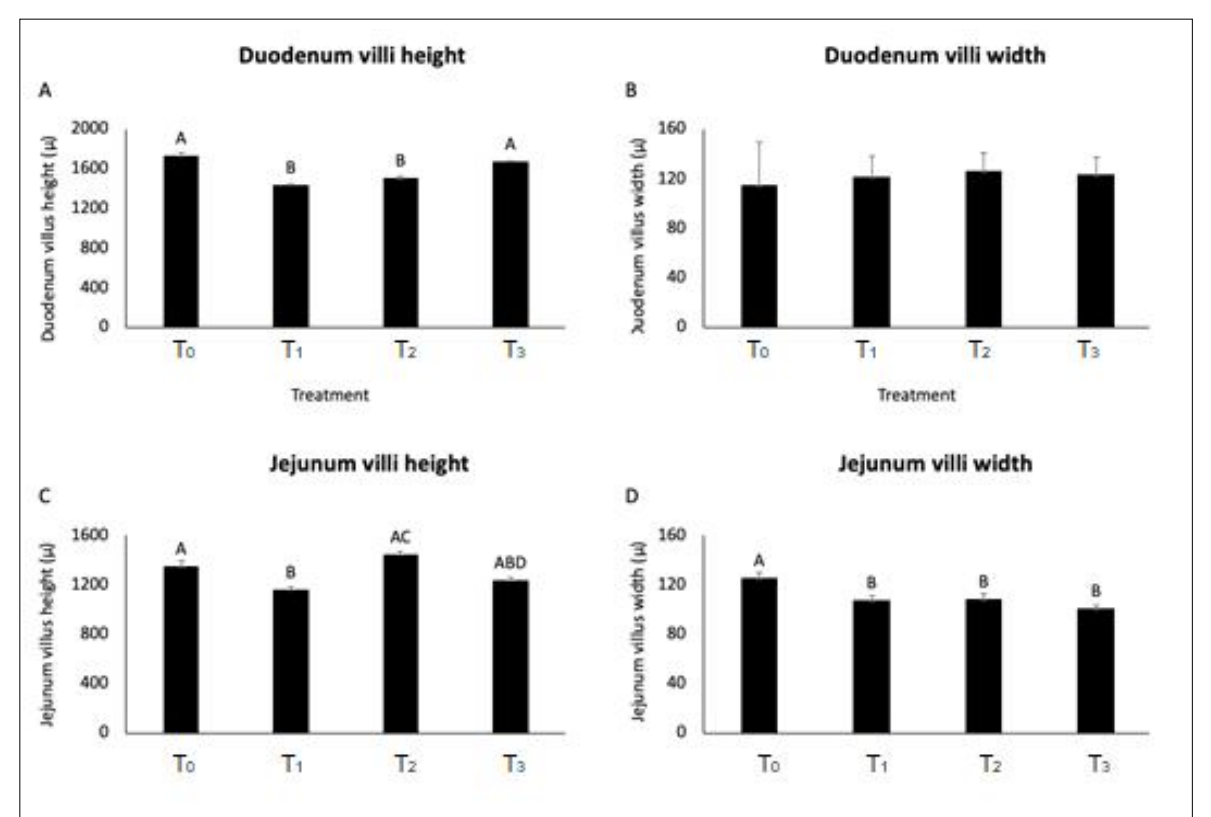

Fig 1: Effect of supplementing the diet with different percent of purple garlic on villi morphometry of the duodenum and jejunum (mean \pm SEM). Means with the same superscripts are not significantly different $(P>0.05)$.

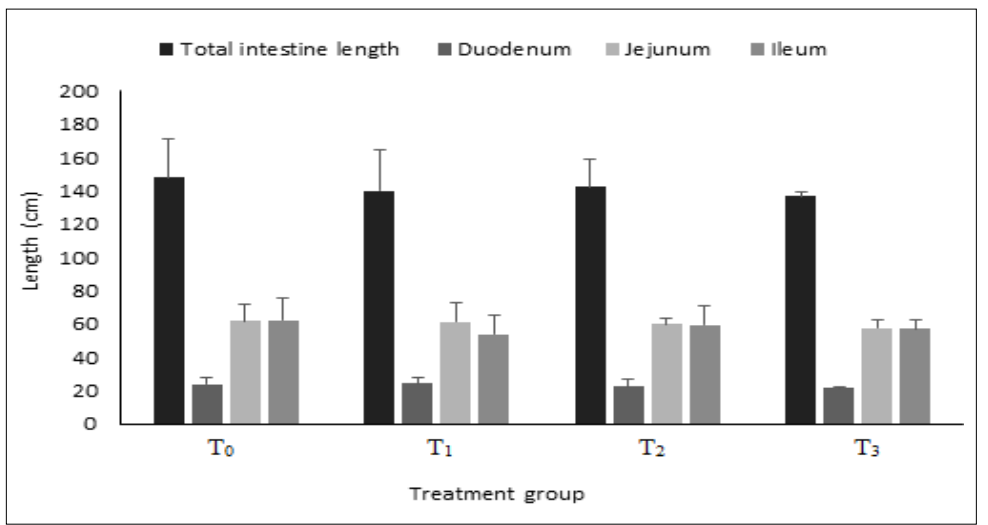

Fig 2: Effect of supplementing the diet with different percent of purple garlic on length $(\mathrm{cm})$ of the duodenum, jejunum and ileum (mean \pm SEM). There are no significant differences between groups $(P>0.05)$.

commercial product) supplemented in the feed of broilers in a daily concentration of $0.2-0.3 \%$ as a natural alternative and viable tool in broilers for growth promotion.

The type and presentation of the garlic used may be related to the results obtained. A standardized commercial product of $65 \%$ Spanish purple garlic powder was used in the present study. In other studies, garlic bulbs purchased from the local market have been used or supplements. It is recognized that different garlic ecotypes and variations in processing could alter the chemical form of the biologically active components (Dubey et al. 2010; Singh, et al. 2014; Singh, 2015). The garlic ecotype "Morado de Las Pedroñeras" showed numerous differences with black garlic in the volatile profile, ascorbic acid content, sugar and polyphenols, antioxidant capacity and composition of phenolic acids and flavonoids (Martínez-Casas et al., 2017). In addition, the highly unstable thiosulfinates, such as allicin (considered responsible for most of the pharmacological activity of crushed raw garlic cloves), disappear during processing and are quickly transformed into various organosulfur components (Lawson and Hunsaker, 2018).

As mentioned previously, a factor that potentially influences garlic's growth performance is gender (Javandel et al., 2008). However, garlic's exact mechanism of action as a growth promotor is uncertain.

\section{CONCLUSION}

Purple garlic supplemented at a dose rate of 0.2 and $0.3 \%$ in the broiler diet has a significant growth promoter in male broilers.

\section{ACKNOWLEDGEMENT}

We thank the companies JR SUAREZ MONEDERO S.L. (Zooallium) for the partial financing to carry out this work. 
The Effect of Supplementing the Diet with Purple Garlic (Allium sativum) on Productivity Variables and Intestinal Morphometry...

We thank CEIEPAv-FMVZ-UNAM, PhD. Pilar Pérez, MSc. Ernesto Ávila and Dr. Arturo Cortes for the support received.

\section{Conflict of interest: None.}

\section{REFERENCES}

Al-Shuwaili, M.A., Ibrahim, E.I. and Al-Bayati, N.M.T. (2015). Effect of dietary herbal plants supplement in turkey diet on performance and some blood biochemical parameters. Global Journal Bioscience and Biotechnology. 4(2): 153157.

Arce, M.J., Ávila, G.E. and López, C.C. (2008). Comportamiento productivo y cambios morfológicos en vellosidades intestinales del pollo de engorda a 21 días de edad con el uso de paredes celulares del Saccharomyces cerevisiae. Veterinary México. 39 (2): 223-228.

Choi, I.H., Park, W.Y. and Kim, Y.J. (2010). Effects of dietary garlic powder and a-tocopherol supplementation on performance, serum cholesterol levels and meat quality of chicken. Poultry Science. 89: 1724-1731.

Dubey, B.K., Singh, R.K. and Bhonde, S.R. (2010).Variability and selection parameters for yield and yield contributing traits in garlic (Allium sativum). Indian Journal of Agricultural Sciences. 80(8): 737-741

EFSA. European Food Safety Autority. (2019). Slaughter of animals: poultry. EFSA Journal 17(11)e05849. https://doi.org/10.2903/ j.efsa.2019.5849. https://efsa.onlinelibrary.wiley.com/doi/ full/10.2903/j.efsa.2019.5849. [Accessed on September 25, 2021).

Javandel, F., Navidshad, B., Seifdavati, J., Pourrahimi, G.H. and Baniyaghoub, S. (2008). The favorite dosage of garlic meal as a feed additive in broiler chickens ratios. Pakistan Journal of Biological Sciences. 11:1746-1749.

Karangiya, V.K., Savsani, H.H., Patil, S.S., Garg, D.D., Murthy, K.S., Ribadiya, N.K. and Vekariya, S.J. (2016). Effect of dietary supplementation of garlic, ginger and their combination on feed intake, growth performance and economics in commercial broilers. Veterinary World. 9(3): 245-250.

Lawson, L.D. and Hunsaker, S.M. (2018). Allicin bioavailability and bioequivalence from garlic supplements and garlic Foods. Nutrients. 10(7): 812 .
Lee, K.C., Kil, D.Y. and Sul, W.J. (2017). Cecal microbiome divergence of broiler chickens by sex and body weight. Journal of Microbiol. 55 939-945.

Martínez-Casas, L., Lage-Yusty, M. and López-Hernández, J. (2017). Changes in the aromatic profile, sugars and bioactive compounds when purple garlic is transformed into black garlic. Journal of Agricultural and Food Chemistry. 65(49): 10804-10811.

Meluzzi, A. and Sirri, F. (2009). Welfare of broiler chickens. Ital. J. Anim. Sci. 8: sup1: 161-173,

Oladele, O.A., Emikpe, B.O. and Bakare, H. (2012). Effects of dietary garlic (Allium sativuml Linn.) supplementation on body weight and gut morphometry of commercial broilers. International Journal of Morphology. 30(1): 238-240.

Pérez-Ortiz, J.M., Galan-Moya, E.M., de la Cruz-Morcillo, M.A, Rodriguez, J.F., Gracia, I. and Garcia, M.T., RedondoCalvo, F.J. (2020). Effective Use of a ThiosulfinateEnriched Allium sativum Extract in Combination with Chemotherapy in Colon Cancer. International Journal of Molecular Science. 21(8): 2766.

Puvaca, N., Kostadinovic, L.J., Ljubojevic, D., Lukac, D., Popovic, S., Dokmanovc, B. and Stanacev, V.S. (2014). Effects of dietary garlic addition on productive performance and blood lipid profile of broiler chickens. Biotechnology in Animal Husbandry. 30(4): 669-676.

Singh, S. (2015). Planting row arrangement and nutrient management in geranium (Pelargonium graveolens) garlic (Allium sativum) intercropping. Indian Journal of Agricultural Research. 49(5): 407-413.

Singh, S., Ram, M., and Yadav, N. (2014). Effect of phosphorus and zinc application on the growth and yield of Geranium (Pelargonium graveolens) intercropped with garlic (Allium sativum). Indian Journal of Agricultural Research. 48(1): 1-8.

Svihus, B. (2014). Function of the digestive system. Journal of Applied Poultry Research. 23: 306-314.

Varlamov, O., Bethea, C.L. and Roberts Jr, C.T. (2015). Sex-specific differences in lipid and glucose metabolism. Frontiers in Endocrinology (Lausanne). 5: 241. doi: 10.3389/ fendo.2014.00241.ss. 